\title{
Decay of Classical Yang-Mills Fields *
}

\author{
R. T. Glassey ${ }^{1}$ and W. A. Strauss ${ }^{2}$ \\ ${ }^{1}$ Department of Mathematics, Indiana University, Bloomington, Indiana 47401, USA \\ ${ }^{2}$ Department of Mathematics, Brown University, Providence, Rhode Island 02912, USA
}

\begin{abstract}
The classical Yang-Mills equations in four-dimensional Minkowski space are invariant under the conformal group. The resulting conservation laws are explicitly exhibited in terms of the Cauchy data at a fixed time. In particular, it is shown that, for any finite-energy solution of the Yang-Mills equations, the local energy tends to zero as $t \rightarrow \infty$.
\end{abstract}

\section{Introduction}

Since the conformal group is 15-dimensional, Noether's theorem implies that there must exist 15 independent conservation laws for the Yang-Mills equations [13]. Ten of them are the familiar laws of conservation of energy, momentum and angular momentum. One is the dilation law due to scale invariance and the remaining four are the inversional laws.

From the first inversional law comes the major decay result. More precisely, for any smooth solution for which the $r F^{\mu v}$ are square integrable, the energy within any cone, which expands at a strictly slower speed than that of a light cone, decays to zero at the rate $t^{-2}$ as $t \rightarrow \infty$. Moreover, for any finite-energy solution, the energy within such a cone tends to zero as $t \rightarrow \infty$. This can be interpreted as stating that all the energy of a solution radiates out along the light cone; that is, at characteristic speed. In particular, there are no "classical lumps". Earlier results asserted that the energy within a fixed sphere tends to zero for some sequence of times $t_{n} \rightarrow \infty[1,4,11]$ and that the radius of gyration moves at characteristic speed [2]. The exact analogue of our result was first derived for the linear wave equation in $[5,14]$ and for the nonlinear wave equation $\square u=u^{3}$ in [9].

The conservation laws are exhibited in terms of the Cauchy data at fixed times, as is appropriate in the study of the existence and asymptotic behavior of solutions. An exposition of these ideas in the case of the nonlinear Klein-Gordon equation may be found in [10]. A similar program of deriving conservation laws is

* $\quad$ Research supported in part by NSF grants MCS 77-01340 and MCS 78-03567 
carried out in the case of the Maxwell-Dirac equations and the Klein-GordonDirac equations in [3]. The ideas in [10] show clearly that useful asymptotic information can also be derived for many systems which are not conformally invariant. In particular, the systems studied in [12] enjoy the $t^{-2}$ decay rate, but we have not carried out the calculations.

In Sect. 2 we list our notational conventions. We have intentionally made an effort to write all the equations explicitly using only standard calculus notation. Section 3 contains the 15 conservation laws. In Sect. 4 we present the decay result. We also derive some "cone estimates". These estimates are bounds for certain quadratic expressions in the field strengths integrated over light cones. Such estimates are crucial ingredients in the scattering theory of the nonlinear KleinGordon equation [6]. In Sect. 5 we consider two special gauges in which the potentials $A^{\mu}$ are shown to have appropriately bounded square integrals. We also explicitly write the 15 conservation laws in terms of potentials in these gauges; these explicit expressions could prove to be useful in a future analysis.

In this paper we do not discuss the question of the existence of solutions. That has been done recently by Segal [8]. Using the quasi-linear character of the equations, he shows that solutions to the Cauchy Problem exist and are smooth locally in time, if the initial data are smooth. If one could prove that all the second derivatives, for instance, of the potentials, had a priori bounded square integrals, the global existence of solutions would follow. In [8] it is shown that a weak limit of solutions of certain truncated equations exists globally. For an exposition of such existence questions in the context of the nonlinear Klein-Gordon equation, see [10]. In the present paper we simply assume that a smooth solution of the Yang-Mills equations is given in all space-time.

\section{The Yang-Mills Equations}

For convenience we choose the gauge group to be $S U(2)$ and consider the unknowns $A^{\mu}(x, t)(\mu=0,1,2,3)$ as real three-vectors, following [7]. The components of each $A^{\mu}$ are written as $A_{k}^{\mu}, k=1,2,3$. The physical variables are $x^{\mu}=\left(x^{0}, x^{1}, x^{2}, x^{3}\right)$, with $x^{0} \equiv t$. We write $x_{\mu}=g_{\mu \nu} x^{v}$ (sum on $v$ ) where the metric $g_{\mu \nu}$ has signature -+++ . Thus $x_{\mu}=\left(x_{0}, x_{1}, x_{2}, x_{3}\right)=\left(-x^{0}, x^{1}, x^{2}, x^{3}\right)$. We further define

$$
\partial^{\mu} \equiv \frac{\partial}{\partial x_{\mu}} ; \quad \delta_{i j}=\left\{\begin{array}{lll}
1 & \text { if } & i=j \\
0 & & i \neq j
\end{array}\right.
$$

and denote by $\varepsilon_{i j k}$ the standard permutation symbol $\left(\varepsilon_{i j k}=0\right.$ if two indices are equal; $\varepsilon_{i j k}=+1$ (resp. -1 ) if $i, j, k$ is an even (resp. odd) permutation of $1,2,3$ ).

The Yang-Mills field strengths are defined by

$$
F_{i}^{\mu v}=\partial^{\mu} A_{i}^{v}-\partial^{v} A_{i}^{\mu}+g \sum_{j, k=1}^{3} \varepsilon_{i j k} A_{j}^{\mu} A_{k}^{v}
$$

where $g$ is a positive constant and $i=1,2,3$. In vector notation, (1) can be written as

$$
\begin{aligned}
& F^{\mu v}=\partial^{\mu} A^{v}-\partial^{v} A^{\mu}+g A^{\mu} \times A^{v} \\
& (\mu, v=0,1,2,3)
\end{aligned}
$$


where $A \times B$ denotes the usual cross product of two three-vectors. We also employ the notation $A \cdot B$ for the ordinary dot product of two such vectors. Now define a differential operator by

$$
\begin{aligned}
& D_{i j}^{v}=\delta_{i j} \partial^{v}+g \sum_{k=1}^{3} \varepsilon_{i k j} A_{k}^{v} \\
& (v=0,1,2,3 ; i, j=1,2,3) .
\end{aligned}
$$

Then the Yang-Mills equations take the form

$$
\begin{aligned}
& -\sum_{j=1}^{3} D_{i j}^{0} F_{j}^{\mu 0}+\sum_{j=1}^{3} \sum_{v=1}^{3} D_{i j}^{v} F_{j}^{\mu \nu}=0 \\
& (\mu=0,1,2,3 ; i=1,2,3) .
\end{aligned}
$$

For convenience, we employ throughout the rest of the paper the following conventions: all sums $\sum_{k}$ are taken over the indices $k=1,2,3$. The physical variables will be denoted by $t, x_{1}, x_{2}, x_{3}$.

We define the analogues of the electric and magnetic field strengths by

$$
E^{k}=F^{k 0} \quad(k=1,2,3)
$$

and

$$
H^{1}=F^{32}, H^{2}=F^{13}, H^{3}=F^{21} .
$$

Let $E$ be the $3 \times 3$ matrix whose columns are $E^{1}, E^{2}$ and $E^{3}$. Let $H$ be the $3 \times 3$ matrix whose columns are $H^{1}, H^{2}$ and $H^{3}$. In this notation the Yang-Mills equations take the following explicit form.

$$
\begin{aligned}
& \frac{\partial H^{1}}{\partial t}=\frac{\partial E^{2}}{\partial x_{3}}-\frac{\partial E^{3}}{\partial x_{2}}+g E^{3} \times A^{2}+g A^{3} \times E^{2}+g A^{0} \times H^{1} \\
& \frac{\partial H^{2}}{\partial t}=\frac{\partial E^{3}}{\partial x_{1}}-\frac{\partial E^{1}}{\partial x_{3}}+g E^{1} \times A^{3}+g A^{1} \times E^{3}+g A^{0} \times H^{2} \\
& \frac{\partial H^{3}}{\partial t}=\frac{\partial E^{1}}{\partial x_{2}}-\frac{\partial E^{2}}{\partial x_{1}}+g E^{2} \times A^{1}+g A^{2} \times E^{1}+g A^{0} \times H^{3} ; \\
& \frac{\partial E^{1}}{\partial t}=\frac{\partial H^{3}}{\partial x_{2}}-\frac{\partial H^{2}}{\partial x_{3}}+g H^{2} \times A^{3}+g A^{2} \times H^{3}+g A^{0} \times E^{1} \\
& \frac{\partial E^{2}}{\partial t}=\frac{\partial H^{1}}{\partial x_{3}}-\frac{\partial H^{3}}{\partial x_{1}}+g H^{3} \times A^{1}+g A^{3} \times H^{1}+g A^{0} \times E^{2} \\
& \frac{\partial E^{3}}{\partial t}=\frac{\partial H^{2}}{\partial x_{1}}-\frac{\partial H^{1}}{\partial x_{2}}+g H^{1} \times A^{2}+g A^{1} \times H^{2}+g A^{0} \times E^{3} ; \\
& \frac{\partial A^{k}}{\partial t}=E^{k}-\frac{\partial A^{0}}{\partial x_{k}}-g A^{k} \times A^{0} \quad(k=1,2,3) ;
\end{aligned}
$$


with the "constraints"

$$
\left\{\begin{array}{l}
\sum_{k}\left(\frac{\partial E^{k}}{\partial x_{k}}+g A^{k} \times E^{k}\right)=0, \\
\sum_{k}\left(\frac{\partial H^{k}}{\partial x_{k}}+g A^{k} \times H^{k}\right)=0 .
\end{array}\right.
$$

\section{The Conservation Laws}

The energy is obtained as follows. Multiply (take the dot product of) (6.j) by $H^{j}$ and (7.j) by $E^{j}(j=1,2,3)$ and add the resulting six equations. The twelve cubic terms, each of which is a scalar triple product, all cancel. The twelve quadratic terms combine to give the energy identity

$$
\frac{\partial}{\partial t} e(E, H)=\sum_{k} \frac{\partial p^{k}}{\partial x_{k}}
$$

where

$$
e(E, H)=\frac{1}{2}\left(|E|^{2}+|H|^{2}\right)=\frac{1}{2} \sum_{k}\left(\left|E^{k}\right|^{2}+\left|H^{k}\right|^{2}\right)
$$

and

$$
\begin{aligned}
& p^{1}=H^{2} \cdot E^{3}-E^{2} \cdot H^{3} \\
& p^{2}=H^{3} \cdot E^{1}-E^{3} \cdot H^{1} \\
& p^{3}=H^{1} \cdot E^{2}-E^{1} \cdot H^{2}
\end{aligned}
$$

Of course if (e) is integrated over all space and the solution vanishes at infinity (which we always assume), we get the conservation of energy:

$$
\int e(E, H) d x=\text { const. }
$$

Unless otherwise specified, the symbol $\int d x$ denotes integration over all of 3-space.

The momenta are obtained as follows. The expression $\partial p^{1} / \partial t$ is the sum of four terms. Use Eqs. (6.2), (6.3), (7.2) and (7.3) to substitute for the time derivatives. Upon adding and combining terms, we obtain

$$
\begin{aligned}
\frac{\partial p^{1}}{\partial t} & =\frac{\partial}{\partial x_{1}} \frac{1}{2}\left[\left|E^{2}\right|^{2}+\left|E^{3}\right|^{2}+\left|H^{2}\right|^{2}+\left|H^{3}\right|^{2}\right] \\
& -\frac{\partial}{\partial x_{2}}\left[H^{1} \cdot H^{2}+E^{1} \cdot E^{2}\right]-\frac{\partial}{\partial x_{3}}\left[H^{1} \cdot H^{3}+E^{1} \cdot E^{3}\right] \\
& +\left(\frac{\partial E^{2}}{\partial x_{2}}+\frac{\partial E^{3}}{\partial x_{3}}\right) \cdot E^{1}+\left(\frac{\partial H^{2}}{\partial x_{2}}+\frac{\partial H^{3}}{\partial x_{3}}\right) \cdot H^{1} \\
& +g E^{2} \cdot\left(E^{1} \times A^{2}\right)+g E^{3} \cdot\left(E^{1} \times A^{3}\right) \\
& +g H^{2} \cdot\left(H^{1} \times A^{2}\right)+g H^{3} \cdot\left(H^{1} \times A^{3}\right) .
\end{aligned}
$$


If Eqs. (9) are now used, the last three lines greatly simplify and we obtain the momentum conservation law. The result is

$$
\frac{\partial p^{j}}{\partial t}=\frac{\partial e}{\partial x_{j}}-\sum_{k} \frac{\partial}{\partial x_{k}}\left(E^{j} \cdot E^{k}+H^{j} \cdot H^{k}\right) \quad(j=1,2,3) .
$$

Thus conservation of momentum is expressed by $\int p^{j}(E, H) d x=$ const.

The other eleven conservation laws are direct consequences of Eqs. (e) and $\left(\mathrm{p}^{j}\right)$. The angular momenta are the integrals of $x_{2} p^{1}-x_{1} p^{2}, x_{3} p^{2}-x_{2} p^{3}, x_{1} p^{3}-x_{3} p^{1}$, and $x_{j} e+t p^{j}(j=1,2,3)$. The dilation identity (scale invariance) gives

$$
\int\left[t e(E, H)+\sum_{k} x_{k} p^{k}(E, H)\right] d x=\text { const. }
$$

The first inversional identity is obtained as follows. Multiply (e) by $r^{2}+t^{2}$ $(r=|x|)$ to obtain

$$
\frac{\partial}{\partial t}\left[\left(r^{2}+t^{2}\right) e\right]-2 t e=\sum_{k} \frac{\partial}{\partial x_{k}}\left[\left(r^{2}+t^{2}\right) p^{k}\right]-2 \sum_{k} x_{k} p^{k} .
$$

Multiply equation $\left(\mathrm{p}^{j}\right)$ by $2 t x_{j}$ to obtain

$$
\begin{aligned}
\frac{\partial}{\partial t}\left[2 t x_{j} p^{j}\right]-2 x_{j} p^{j}=\frac{\partial}{\partial x_{j}}\left[2 t x_{j} e\right] & -\sum_{k} \frac{\partial}{\partial x_{k}}\left[2 t x_{j}\left(E^{j} \cdot E^{k}+H^{j} \cdot H^{k}\right)\right] \\
& +2 t\left(\left|E^{j}\right|^{2}+\left|H^{j}\right|^{2}\right)-2 t e
\end{aligned}
$$

$(j=1,2,3)$. Summing the resulting four equations, we obtain

$$
\frac{\partial}{\partial t}\left[\left(r^{2}+t^{2}\right) e+2 t \sum_{j} x_{j} p^{j}\right]=\sum_{k} \frac{\partial n^{k}}{\partial x_{k}}
$$

where

$$
n^{k}=\left(r^{2}+t^{2}\right) p^{k}+2 t x_{k} e-2 t \sum_{j} x_{j}\left(E^{j} \cdot E^{k}+H^{j} \cdot H^{k}\right) .
$$

Finally, the last three inversional conservation laws are

$$
\begin{aligned}
& \int\left[t x_{j} e(E, H)+\frac{1}{2}\left(t^{2}-r^{2}\right) p^{j}(E, H)+\sum_{m} x_{j} x_{m} p^{m}(E, H)\right] d x \\
& \quad=\text { const. } \quad(j=1,2,3) .
\end{aligned}
$$

\section{Estimations}

The fundamental bound is that of the energy:

$$
\sum_{k} \int\left(\left|E^{k}\right|^{2}+\left|H^{k}\right|^{2}\right) d x=\text { const. }
$$

That is, all of the 18 components of $E$ and $H$ have square integrals which are bounded in time.

Next, let $K$ be any light cone. Then we claim that 12 of the 18 components are square-integrable on $K$. These 12 components vary from point to point on $K$. 
Suppose, for instance, that $K$ is the positive light cone $\{|x|=t\}$. Integrate (e) over the 4-dimensional region $\{|x|<t<T\}$. Then

$$
\int_{|x|=t<T}\left(\frac{1}{2}|E|^{2}+\frac{1}{2}|H|^{2}+\sum_{k} \omega_{k} p^{k}\right) \frac{d S}{\sqrt{2}}=\frac{1}{2} \int_{|x|<t=T}\left(|E|^{2}+|H|^{2}\right) d x
$$

where $\omega_{k}=\frac{x_{k}}{r}$ and $d S$ denotes the usual measure on the surface of $K$. Letting $T \rightarrow \infty$, we obtain

$$
\int_{K}\left(\frac{1}{2}|E|^{2}+\frac{1}{2}|H|^{2}+\sum_{k} \omega_{k} p^{k}\right) d S \leqq \frac{1}{\sqrt{2}} \int\left(|E|^{2}+|H|^{2}\right) d x=\text { const. }
$$

The integrand on the left is nonnegative and can be written as a sum of squares. For this purpose we introduce the following notation. Recall that $E$ denotes the matrix whose columns are $E^{1}, E^{2}$ and $E^{3}$, and $H$ the matrix whose columns are $H^{1}$, $H^{2}$ and $H^{3}$. Now $E^{T}$ is the transpose of $E$ and $|E|^{2}=\operatorname{tr}\left(E^{T} E\right)=\sum_{i, j}\left|E_{i}^{j}\right|^{2}$.

Let $\omega$ be the vector $\frac{x}{|x|}$. Given $\omega$ with $|\omega|=1$, we introduce unit vectors $\alpha, \beta$ such that $\alpha, \beta, \omega$ form an orthonormal basis for $\mathbb{R}^{3}$ with $\alpha \times \beta=\omega$. We then have

$$
|E|^{2}=|E \omega|^{2}+|E \alpha|^{2}+|E \beta|^{2}
$$

and

$$
|H|^{2}=|H \omega|^{2}+|H \alpha|^{2}+|H \beta|^{2}
$$

since $\alpha, \beta, \omega$ are orthonormal. Next, we calculate

$$
\begin{aligned}
\omega_{1} p^{1} & =\left(\alpha_{2} \beta_{3}-\alpha_{3} \beta_{2}\right)\left(H^{2} \cdot E^{3}-E^{2} \cdot H^{3}\right) \\
& =\alpha_{2} \beta_{3}\left(H^{2} \cdot E^{3}-H^{3} \cdot E^{2}\right)+\alpha_{3} \beta_{2}\left(H^{3} \cdot E^{2}-H^{2} \cdot E^{3}\right) .
\end{aligned}
$$

Hence we can write

$$
\sum_{k} \omega_{k} p^{k}=\sum_{j, k} \alpha_{j} \beta_{k}\left(H^{j} \cdot E^{k}-H^{k} \cdot E^{j}\right)=H \alpha \cdot E \beta-H \beta \cdot E \alpha .
$$

It follows that the integrand on the left above can be written as

$$
\begin{aligned}
& \frac{1}{2}|E|^{2}+\frac{1}{2}|H|^{2}+\sum_{k} \omega_{k} p^{k} \\
& \quad=\frac{1}{2}|E \omega|^{2}+\frac{1}{2}|H \omega|^{2}+\frac{1}{2}|E \alpha-H \beta|^{2}+\frac{1}{2}|E \beta+H \alpha|^{2} .
\end{aligned}
$$

Thus the twelve components of the vectors $E \omega, H \omega, E \alpha-H \beta$, and $E \beta+H \alpha$ are square-integrable on $K$.

Next we integrate (13) over all space to obtain

$$
\int\left[\left(r^{2}+t^{2}\right) e(E, H)+2 \operatorname{tr} \sum_{k} \omega_{k} p^{k}(E, H)\right] d x=\text { const. }
$$

where $\omega_{k}=\frac{x_{k}}{r}$. The calculation just completed shows that $\left|\sum_{k} \omega_{k} p^{k}\right| \leqq e$. Hence this conservation law implies

$$
\int(t-r)^{2} e(E, H) d x \leqq \text { const. }
$$


In particular, if we restrict the integral to a cone of smaller aperture than a light cone, we get

$$
(\varepsilon t-R)^{2} \int_{|x|<R+(1-\varepsilon) t} e(E, H) d x \leqq \text { const. }
$$

for $t>R \varepsilon^{-1}$. Therefore we have proved the following

Theorem. Let $R>0$ and $0<\varepsilon \leqq 1$. Then

$$
\int_{|x|<R+(1-\varepsilon) t}\left[|E|^{2}+|H|^{2}\right] d x=O\left(t^{-2}\right) \text { as } t \rightarrow \infty .
$$

This is valid for solutions whose Cauchy data (at any time) satisfies $\int r^{2} e(E, H) d x$ $<\infty$.

We can extend the class of solutions for which a decay result is valid as follows : Let $E, H, A$ be any finite energy solution of (6)-(9). Thus it is only assumed that

$$
\int\left(|E|^{2}+|H|^{2}\right) d x<\infty
$$

at some fixed time. Let $\zeta_{n}(x)$ be the standard cut-off function: $\zeta_{n} \in C^{\infty}, 0 \leqq \zeta_{n} \leqq 1$, $\zeta_{n}(x) \equiv 1$ for $|x| \leqq n, \zeta_{n}(x) \equiv 0$ for $|x|>n+1$. Consider the solutions $E^{(n)}, H^{(n)}$ whose Cauchy data is obtained from the original Cauchy data by multiplying it by $\zeta_{n}(x)$. We assume that

$$
\sup _{t} \int\left[\left|E^{(n)}-E\right|^{2}+\left|H^{(n)}-H\right|^{2}\right] d x \rightarrow 0 \text { as } n \rightarrow \infty \text {. }
$$

Since the Cauchy data of $E^{(n)}, H^{(n)}$ have compact support, the hypotheses of the theorem are satisfied, and hence $E^{(n)}, H^{(n)}$ enjoy the $t^{-2}$-rate of local energy decay. We employ the notation

$$
\|u\|_{t}^{2} \equiv \int_{|x|<R+(1-\varepsilon) t}|u(x, t)|^{2} d x .
$$

Now let $\delta>0$ be arbitrary. By (16) we can choose $n=N$ such that

$$
\left(\int\left|E-E^{(N)}\right|^{2} d x\right)^{1 / 2}+\left(\int\left|H-H^{(N)}\right|^{2} d x\right)^{1 / 2}<\frac{\delta}{2} .
$$

Then, using the theorem, we choose $T$ so large that

$$
\left\|E^{(N)}\right\|_{t}+\left\|H^{(N)}\right\|_{t}<\frac{\delta}{2}
$$

for all $t \geqq T$. Since $\delta$ was arbitrary, we have the following result:

Corollary 1. Assume that any finite-energy solution can be approximated by cut-off solutions as in (16). Let $R>0,0<\varepsilon \leqq 1$. Then for every finite-energy solution of the Yang-Mills equations we have

$$
\lim _{t \rightarrow \infty} \int_{|x|<R+(1-\varepsilon) t}\left(|E|^{2}+|H|^{2}\right) d x=0 .
$$


Corollary 2. If the Yang-Mills equations possess a finite-energy solution of the form

$$
E=E(x-c t), \quad H=H(x-b t)
$$

where $b$ and $c$ are constant vectors of norm less than one, then $E=H=0$.

Let $K$ again be any light cone, say $|x|=t$. We integrate (13) over a 4-dimensional region as before to obtain

$$
\int_{K}\left[\left(r^{2}+t^{2}\right) e+2 \operatorname{tr} \sum_{k} \omega_{k} p^{k}+\sum_{k} \omega_{k} n^{k}\right] d S \leqq \text { const. }
$$

Using the definition of $n^{k}$, we can write the last integrand as

$$
\begin{aligned}
I & =(t+r)^{2}\left(e+\sum_{k} \omega_{k} p^{k}\right)-2 \operatorname{tr} \sum_{j, k} \omega_{j} \omega_{k}\left(E^{j} \cdot E^{k}+H^{j} \cdot H^{k}\right) \\
& =(t+r)^{2}\left(e+\sum_{k} \omega_{k} p^{k}\right)-2 \operatorname{tr}\left\{\left|\sum_{k} \omega_{k} E^{k}\right|^{2}+\left|\sum_{k} \omega_{k} H^{k}\right|^{2}\right\} \\
& =\frac{(t+r)^{2}}{2}\left[|E \omega|^{2}+|H \omega|^{2}+|E \alpha-H \beta|^{2}+|E \beta+H \alpha|^{2}\right]-2 \operatorname{tr}\left[|E \omega|^{2}+|H \omega|^{2}\right] \\
& =\frac{(t-r)^{2}}{2}\left[|E \omega|^{2}+|H \omega|^{2}\right]+\frac{(t+r)^{2}}{2}\left[|E \alpha-H \beta|^{2}+|E \beta+H \alpha|^{2}\right] .
\end{aligned}
$$

Therefore 6 of the 18 components of $E$ and $H$ are square-integrable on $K$ with the weight function $t^{2}$.

\section{Special Gauges}

It is useful to obtain bounds on the potentials $A^{\mu}$ as well as on the field strengths $E$ and $H$. We begin with the Lorentz gauge

$$
\frac{\partial A^{0}}{\partial t}+\sum_{k} \frac{\partial A^{k}}{\partial x_{k}}=0 .
$$

In this gauge the potentials have appropriately bounded square integrals. Indeed, we take the scalar product of $E^{k}\left[\right.$ Eq. (8)] with $A^{k}$ and sum on $k$ :

$$
\begin{aligned}
\sum_{k} E^{k} \cdot A^{k} & =\sum_{k}\left(\frac{\partial A^{k}}{\partial t}+\frac{\partial A^{0}}{\partial x_{k}}+g A^{k} \times A^{0}\right) \cdot A^{k} \\
& =\frac{\partial}{\partial t} \frac{1}{2} \sum_{k}\left|A^{k}\right|^{2}+\sum_{k} \frac{\partial}{\partial x_{k}}\left(A^{0} \cdot A^{k}\right)-\sum_{k} A^{0} \cdot \frac{\partial A^{k}}{\partial x_{k}} .
\end{aligned}
$$

Using $\left(^{*}\right)$ and integrating over all space, we obtain

$$
\begin{aligned}
\frac{d}{d t} \int\left[\left|A^{0}\right|^{2}+\sum_{k}\left|A^{k}\right|^{2}\right] d x & =2 \int \sum_{k} E^{k} \cdot A^{k} d x \\
& \leqq 2\left(\sum_{k} \int\left|E^{k}\right|^{2} d x\right)^{1 / 2}\left(\sum_{k} \int\left|A^{k}\right|^{2} d x\right)^{1 / 2}
\end{aligned}
$$


whence

$$
\begin{aligned}
& {\left[\int\left(\left|A^{0}\right|^{2}+\sum_{k}\left|A^{k}\right|^{2}\right) d x\right]^{1 / 2}} \\
& \quad \leqq\left[\int\left(\left|A^{0}(x, 0)\right|^{2}+\sum_{k}\left|A^{k}(x, 0)\right|^{2}\right) d x\right]^{1 / 2}+\int_{0}^{t}\left(\int|E|^{2} d x\right)^{1 / 2} d s
\end{aligned}
$$

for all $t$. Therefore

$$
\int\left[\left|A^{0}\right|^{2}+\sum_{k}\left|A^{k}\right|^{2}\right] d x=O\left(1+t^{2}\right)
$$

for all $t$.

In this gauge the Yang-Mills equations take the form

$$
\begin{aligned}
& \frac{\partial^{2} A^{0}}{\partial t^{2}}-\Delta A^{0}+g\left[\frac{\partial A^{0}}{\partial t} \times A^{0}+\sum_{k}\left(2 \frac{\partial A^{0}}{\partial x_{k}} \times A^{k}+\frac{\partial A^{k}}{\partial t} \times A^{k}\right)\right] \\
& +g^{2} \sum_{k}\left\{\left|A^{k}\right|^{2} A^{0}-\left(A^{k} \cdot A^{0}\right) A^{k}\right\}=0 ; \\
& \frac{\partial^{2} A^{j}}{\partial t^{2}}-\Delta A^{j}+g\left[2 \frac{\partial A^{j}}{\partial t} \times A^{0}+\frac{\partial A^{0}}{\partial x_{j}} \times A^{0}+\sum_{k}\left(A^{k} \times \frac{\partial A^{k}}{\partial x_{j}}+2 \frac{\partial A^{j}}{\partial x_{k}} \times A^{k}\right)\right] \\
& +g^{2}\left[\sum_{k}\left\{\left|A^{k}\right|^{2} A^{j}-\left(A^{k} \cdot A^{j}\right) A^{k}\right\}-\left|A^{0}\right|^{2} A^{j}+\left(A^{0} \cdot A^{j}\right) A^{0}\right]=0 .
\end{aligned}
$$

We have made use of the identity

$$
A \times(B \times C)=(C \cdot A) B-(B \cdot A) C .
$$

The 15 conformal conservation laws now take the following forms, after some tedious computations.

The energy density is

$$
\begin{aligned}
-2 e(A)= & \left|\frac{\partial A^{0}}{\partial t}\right|^{2}+\left|\nabla A^{0}\right|^{2}-\sum_{k}\left(\left|\frac{\partial A^{k}}{\partial t}\right|^{2}+\left|\nabla A^{k}\right|^{2}\right) \\
& +2 g \sum_{k}\left\{\frac{\partial A^{0}}{\partial x_{k}} \cdot A^{k} \times A^{0}+\sum_{n} A^{k} \cdot A^{n} \times \frac{\partial A^{k}}{\partial x_{n}}\right\} \\
& +g^{2} \sum_{k}\left|A^{k} \times A^{0}\right|^{2}-\frac{g^{2}}{2} \sum_{k, n}\left|A^{k} \times A^{n}\right|^{2} .
\end{aligned}
$$

The momentum densities are $p^{j}(A)=-\frac{\partial A^{0}}{\partial t} \cdot \frac{\partial A^{0}}{\partial x_{j}}+\sum_{k}\left(\frac{\partial A^{k}}{\partial t} \cdot \frac{\partial A^{k}}{\partial x_{j}}-g \frac{\partial A^{k}}{\partial x_{j}} \cdot A^{0} \times A^{k}\right)$ $(j=1,2,3)$. 
Notice that $e(A)$ and $p^{j}(A)$ differ from $e$ and $p^{j}$ of Sect. 3 by certain $x$-derivatives (which integrate to zero). The angular momenta are

$$
\begin{aligned}
& \int\left[A^{j} \cdot \frac{\partial A^{0}}{\partial t}-A^{0} \cdot \frac{\partial A^{j}}{\partial t}+x_{j} e(A)+t p^{j}(A)\right] d x=\text { const. } \\
& (j=1,2,3)
\end{aligned}
$$

and

$$
\begin{aligned}
& \int\left[-x_{l} p^{m}(A)+x_{m} p^{l}(A)+A^{l} \cdot \frac{\partial A^{m}}{\partial t}-A^{m} \cdot \frac{\partial A^{l}}{\partial t}+2 g A^{0} \cdot A^{l} \times A^{m}\right] d x \\
& =\text { const. } \quad(l \neq m, l=1,2,3) .
\end{aligned}
$$

The dilational identity is

$$
\begin{aligned}
\int\left[A^{0} \cdot \frac{\partial A^{0}}{\partial t}\right. & -\sum_{k} A^{k} \cdot \frac{\partial A^{k}}{\partial t}-t e(A)+r \frac{\partial A^{0}}{\partial r} \cdot \frac{\partial A^{0}}{\partial t}-\sum_{k} r \frac{\partial A^{k}}{\partial r} \cdot \frac{\partial A^{k}}{\partial t} \\
& \left.+g \sum_{k} r \frac{\partial A^{k}}{\partial r} \cdot A^{0} \times A^{k}\right] d x=\text { const. }
\end{aligned}
$$

The inversional identities are

$$
\begin{aligned}
\int\left[-\left(r^{2}+t^{2}\right) e(A)+2 t\left(A^{0} \cdot \frac{\partial A^{0}}{\partial t}-\sum_{k} A^{k} \cdot \frac{\partial A^{k}}{\partial t}\right)\right. \\
+2 t r\left(\frac{\partial A^{0}}{\partial t} \cdot \frac{\partial A^{0}}{\partial r}-\sum_{k} \frac{\partial A^{k}}{\partial t} \cdot \frac{\partial A^{k}}{\partial r}\right) \\
+2 g t \sum_{k} r \frac{\partial A^{k}}{\partial r} \cdot A^{0} \times A^{k}-3\left|A^{0}\right|^{2}+\sum_{k}\left|A^{k}\right|^{2} \\
\left.+2 \sum_{k} x_{k}\left(A^{0} \cdot \frac{\partial A^{k}}{\partial t}-A^{k} \cdot \frac{\partial A^{0}}{\partial t}\right)\right] d x=\text { const. }
\end{aligned}
$$

and

$$
\begin{aligned}
\int\left[x_{j}\right. & \left(A^{0} \cdot \frac{\partial A^{0}}{\partial t}-\sum_{k} A^{k} \cdot \frac{\partial A^{k}}{\partial t}\right)+t\left(A^{0} \cdot \frac{\partial A^{j}}{\partial t}-A^{j} \cdot \frac{\partial A^{0}}{\partial t}\right) \\
& -t x_{j} e(A)-A^{0} \cdot A^{j}+\frac{1}{2}\left(r^{2}-t^{2}\right) p^{j}(A) \\
& \left.+\sum_{m} x_{m}\left\{-x_{j} p^{m}(A)+A^{j} \cdot \frac{\partial A^{m}}{\partial t}-A^{m} \cdot \frac{\partial A^{j}}{\partial t}+2 g A^{0} \cdot A^{j} \times A^{m}\right\}\right] d x \\
& =\text { const. } \quad(j=1,2,3) .
\end{aligned}
$$

The Lorentz gauge has the drawback that some of the quadratic terms in the energy density $e(A)$ are positive and some are negative. Consider instead the Coulomb gauge

$$
\sum_{k} \frac{\partial A^{k}}{\partial x_{k}}=0 .
$$


By the same method as we obtained (17), we have

$$
\left(\int \sum_{k}\left|A^{k}(x, t)\right|^{2} d x\right)^{1 / 2} \leqq\left(\int \sum_{k}\left|A^{k}(x, 0)\right|^{2} d x\right)^{1 / 2}+\int_{0}^{t}\left(\int|E|^{2} d x\right)^{1 / 2} d s
$$

hence

$$
\int \sum_{k}\left|A^{k}(x, t)\right|^{2} d x=O\left(1+t^{2}\right) \text { for all } t .
$$

In the Coulomb gauge the Yang-Mills equations take the form

$$
\begin{aligned}
& -\Delta A^{0}+g \sum_{k}\left(2 \frac{\partial A^{0}}{\partial x_{k}} \times A^{k}+\frac{\partial A^{k}}{\partial t} \times A^{k}\right) \\
& \quad+g^{2} \sum_{k}\left(\left|A^{k}\right|^{2} A^{0}-\left(A^{k} \cdot A^{0}\right) A^{k}\right)=0 \\
& \frac{\partial^{2} A^{k}}{\partial t^{2}}-\Delta A^{k}+\frac{\partial^{2} A^{0}}{\partial t \partial x_{k}} \\
& +g\left[2 \frac{\partial A^{k}}{\partial t} \times A^{0}+A^{k} \times \frac{\partial A^{0}}{\partial t}-A^{0} \times \frac{\partial A^{0}}{\partial x_{k}}+\sum_{j}\left\{2 \frac{\partial A^{k}}{\partial x_{j}} \times A^{j}+A^{j} \times \frac{\partial A^{j}}{\partial x_{k}}\right\}\right] \\
& +g^{2}\left[-\left|A^{0}\right|^{2} A^{k}+\left(A^{k} \cdot A^{0}\right) A^{0}+\sum_{j}\left\{\left|A^{j}\right|^{2} A^{k}-\left(A^{j} \cdot A^{k}\right) A^{j}\right\}\right]=0
\end{aligned}
$$

$(k=1,2,3)$.

If (19.0) is multiplied by $A^{0}$, the useful identity

$$
\begin{aligned}
\left|\nabla A^{0}\right|^{2}-\nabla \cdot\left(A^{0} \cdot \nabla A^{0}\right) & +g \sum_{k}\left(2 A^{0} \cdot \frac{\partial A^{0}}{\partial x_{k}} \times A^{k}+A^{0} \cdot \frac{\partial A^{k}}{\partial t} \times A^{k}\right) \\
& +g^{2} \sum_{k}\left|A^{k} \times A^{0}\right|^{2}=0
\end{aligned}
$$

is obtained. The energy density takes the form

$$
\begin{aligned}
e(A)= & \frac{1}{2}\left\{\left|\nabla A^{0}\right|^{2}+\sum_{k}\left(\left|\frac{\partial A^{k}}{\partial t}\right|^{2}+\left|\nabla A^{k}\right|^{2}\right)\right\} \\
& +g \sum_{k}\left\{\frac{\partial A^{0}}{\partial x_{k}} \cdot A^{k} \times A^{0}+A^{0} \cdot \frac{\partial A^{k}}{\partial t} \times A^{k}+\sum_{l} \frac{\partial A^{l}}{\partial x_{k}} \cdot A^{k} \times A^{l}\right\} \\
& +\frac{g^{2}}{2} \sum_{k}\left|A^{k} \times A^{0}\right|^{2}+\frac{g^{2}}{4} \sum_{k, l}\left|A^{k} \times A^{l}\right|^{2}
\end{aligned}
$$

with the understanding as before that it may differ from the preceding energy densities by a spatial divergence (which integrates to zero). The Coulomb gauge has the advantage that the energy density can be written so that the quadratic terms are positive.

The momentum densities are

$$
p^{j}(A)=\sum_{k}\left(\frac{\partial A^{k}}{\partial t} \cdot \frac{\partial A^{k}}{\partial x_{j}}+g \frac{\partial A^{k}}{\partial x_{j}} \cdot A^{k} \times A^{0}\right) \quad(j=1,2,3) .
$$


The angular momenta are

$$
\int\left[x_{j} e(A)-A^{0} \cdot \frac{\partial A^{j}}{\partial t}+t p^{j}(A)\right] d x=\text { const. } \quad(j=1,2,3)
$$

and

$$
\begin{aligned}
& \int\left[x_{m} p^{j}(A)-x_{j} p^{m}(A)+A^{j} \cdot \frac{\partial A^{m}}{\partial t}-A^{m} \cdot \frac{\partial A^{j}}{\partial t}+2 g A^{0} \cdot A^{j} \times A^{m}\right] d x=\text { const. } \\
& (m \neq j, j=1,2,3) .
\end{aligned}
$$

The dilational conservation law is

$$
\int\left[t e(A)+\sum_{k}\left\{A^{k} \cdot \frac{\partial A^{k}}{\partial t}+r \frac{\partial A^{k}}{\partial r} \cdot \frac{\partial A^{k}}{\partial t}+g r \frac{\partial A^{k}}{\partial r} \cdot A^{k} \times A^{0}\right\}\right] d x=\text { const. }
$$

The inversional conservation laws are

$$
\begin{aligned}
& \int\left[\left(r^{2}+t^{2}\right) e(A)-\sum_{k}\left|A^{k}\right|^{2}-2 \sum_{k} x_{k} A^{0} \cdot \frac{\partial A^{k}}{\partial t}\right. \\
& \left.\quad+2 t \sum_{k}\left\{A^{k} \cdot \frac{\partial A^{k}}{\partial t}+r \frac{\partial A^{k}}{\partial t} \cdot \frac{\partial A^{k}}{\partial r}+g r \frac{\partial A^{k}}{\partial r} \cdot A^{k} \times A^{0}\right\}\right] d x=\text { const. }
\end{aligned}
$$

and

$$
\begin{aligned}
\int\left[t x_{j} e(A)\right. & -t A^{0} \cdot \frac{\partial A^{j}}{\partial t}+\frac{1}{2}\left(t^{2}-r^{2}\right) p^{j}(A) \\
& +\sum_{m} x_{j} x_{m} p^{m}(A)+A^{0} \cdot A^{j}+\sum_{m} x_{j} A^{m} \cdot \frac{\partial A^{m}}{\partial t} \\
& \left.+\sum_{m} x_{m}\left\{A^{m} \cdot \frac{\partial A^{j}}{\partial t}-A^{j} \cdot \frac{\partial A^{m}}{\partial t}+2 g A^{0} \cdot A^{m} \times A^{j}\right\}\right] d x=\text { const. }
\end{aligned}
$$

$(j=1,2,3)$.

Acknowledgement. We thank R. Peccei for informative discussions.

\section{References}

1. Coleman, S.: There are no classical glueballs. Commun. math. Phys. 55, 113-116 (1977)

2. Coleman,S., Smarr, L.: Are there geon analogues in sourceless gauge-field theories? Commun. math. Phys. 56, 1-9 (1977)

3. Glassey, R., Strauss, W.: Conservation laws for the classical Maxwell-Dirac and Klein-GordonDirac equations. J. Math. Phys. (to appear) (1979)

4. Maag, M.: Some constraints on finite energy solutions in non-Abelian gauge theories. J. Math. Phys. 19, 991-993 (1978)

5. Morawetz,C.: The limiting amplitude principle. Commun. Pure Appl. Math. 15, 349-361 (1962)

6. Morawetz,C., Strauss, W.: Decay and scattering of solutions to a nonlinear relativistic wave equation. Commun. Pure Appl. Math. 25, 1-31 (1972)

7. Peccei,R.: Resolution of ambiguities of non-abelian gauge field theories in the Coulomb gauge. Preprint (1978) 
8. Segal, I.: The Cauchy problem for the Yang-Mills equations. J. Funct. Anal. (to appear)

9. Strauss, W. : La decroissance asymptotique des solutions des équations d'onde non linéaires. C. R. Acad. Sci. 256, 2749-2750 (1963)

10. Strauss, W. : Nonlinear invariant wave equations. In : Lecture notes in physics, Vol. 73, pp. 197-249 (Erice 1977). Berlin, Heidelberg, New York: Springer 1978

11. Weder, R.: Absence of classical lumps. Commun. math. Phys. 57, 161-164 (1977)

12. Weder, R.: Absence of classical lumps in constrained systems. Preprint (1978)

13. Yang, C.N., Mills, R.: Conservation of isotropic spin and isotropic gauge invariance. Phys. Rev. 96, 191-195 (1954)

14. Morawetz, C.: Appendix 3 in Lax, P., Phillips, R.: Scattering theory. New York, London: Academic Press 1967

Communicated by A. Jaffe

Received August 7, 1978

The results of this paper are valid for Yang-Mills fields coupled to scalar fields. 
